\title{
Quality of life of patients with ADPKD- Toranomon PKD QOL study: cross-sectional study
}

\author{
Tatsuya Suwabe*, Yoshifumi Ubara, Koki Mise, Masahiro Kawada, Satoshi Hamanoue, Keiichi Sumida, \\ Noriko Hayami, Junichi Hoshino, Rikako Hiramatsu, Masayuki Yamanouchi, Eiko Hasegawa, Naoki Sawa \\ and Kenmei Takaichi
}

\begin{abstract}
Background: The quality of life (QOL) of patients with autosomal dominant polycystic kidney disease (ADPKD) has not been investigated well. This study was performed to clarify the QOL of patients with ADPKD and to identify factors that affected their QOL.

Methods: The present cross-sectional study is part of a prospective observational study on the QOL of ADPKD patients. Patients with ADPKD who were referred to Toranomon Hospital between March 2010 and November 2012 were enrolled. The short form-36 (SF-36) questionnaire and our original 12-item questionnaire were used to evaluate QOL. We analyzed the results of the questionnaire survey and then investigated correlations between QOL and clinical features.

Results: A total of 219 patients (93 men and 126 women) were enrolled and their mean age was $55.1 \pm 10.8$ years. There were 108 patients on dialysis. The SF-36 scores (PCS, MCS, and RCS) of all patients were significantly lower than the mean scores for the Japanese population. Stepwise multiple regression analysis demonstrated that $\mathrm{Hb}$, serum Alb, ascites, and cerebrovascular disease all had a significant influence on the PCS, while mental disease had a significant influence on the MCS and serum Alb significantly influenced the RCS. The total liver and kidney volume (TLKV) and the dialysis status were not significantly associated with any of the SF-36 scores by multiple regression analysis, but TLKV was closely correlated with abdominal distention and distention had an important influence on QOL. Pain, sleep disturbance, heartburn, fever, gross hematuria, and anorexia also affected QOL, but these variables were not correlated with TLKV.
\end{abstract}

Conclusions: Several factors influence QOL, so improving symptoms unrelated to TLKV as well as reducing abdominal distention can improve the QOL of ADPKD patients.

Keywords: Quality of life, Polycystic kidney disease, Dialysis, Total liver and kidney volume

\section{Background}

Autosomal dominant polycystic kidney disease (ADPKD) is a common disorder that occurs in approximately 1 in every 400 to 1000 live births [1,2]. It is estimated that less than one-half of ADPKD patients will be diagnosed during life because the disease is often clinically silent [1]. However, some patients with ADPKD develop massive enlargement of the kidneys and/or liver. We have performed renal transcatheter arterial embolization (TAE) in 797 symptomatic patients with renal enlargement and hepatic TAE in 325 symptomatic patients with hepatomegaly

\footnotetext{
* Correspondence: suwabe@toranomon.gr.jp

Department of Nephrology, Toranomon Hospital, Tokyo, Japan
}

up to November 2012, and we have previously reported that TAE is an effective method of reducing the hepatic or renal volume [3-6]. Most of our patients who received renal or hepatic TAE had symptoms related to abdominal distension and their quality of life (QOL) was obviously impaired. It has been reported that ADPKD affects the physical condition of patients and also has an impact on mental health [7]. Pain is a common symptom of ADPKD and it has a detrimental effect on QOL $[8,9]$, but the QOL of ADPKD patients and the factors influencing it have not been clarified. Recently, the TEMPO study showed that tolvaptan slows the increase of total kidney volume and the decline of renal function in patients with ADPKD [10], but 
it is still unknown what benefits are related to kidney volume and renal function. Rizk et al. evaluated the QOL of predialysis patients with ADPKD by the short form-36 (SF-36) questionnaire and they reported that such patients did not have worse QOL than the general population [11]. They also reported that the QOL of patients with a mean renal volume over $1000 \mathrm{~cm}^{3}$ was not significantly different from that of patients with a mean volume under $1000 \mathrm{~cm}^{3}$, but they did not assess the influence of hepatic volume. The average renal volume of the patients receiving renal TAE at our hospital was over $2000 \mathrm{~cm}^{3}$ [3], and some of our predialysis patients also had severe hepatomegaly (Figure 1). We considered that the mean renal volume in Rizk's study might have been too small to affect QOL and that hepatic volume should also be taken into account. Therefore, we started to investigate the QOL of ADPKD patients who were referred to our hospital for massive enlargement of the kidneys or liver, employing the SF-36 questionnaire and our original 12-item questionnaire to evaluate QOL. The SF-36 questionnaire is one of the most common methods of evaluating QOL worldwide, as well as in Japan [12-14]. Because mean SF-36 scores for the general Japanese population are available, it is easy to evaluate the QOL of patients by comparison with the general population. We also devised twelve questions of our own to evaluate the specific symptoms of patients with ADPKD.

In the present study, we employed these tools to clarify the QOL of ADPKD patients with massive enlargement of the kidneys and/or liver and to identify factors affecting their QOL.

\section{Methods}

This was a cross-sectional study that was part of a prospective observational study on the QOL of patients with ADPKD. This study was reviewed and approved by the ethics committee of Toranomon Hospital in March 2010. Written informed consent was obtained from each participant before entry into the study, and also for publication of any accompanying images. A copy of the written consent for publication statement is available for review by the Editor of this journal. This study was registered with the University Hospital Medical Information Network (UMIN) as "Quality of life (QOL) of patients with autosomal dominant polycystic kidney disease (ADPKD): Influence of renal TAE or hepatic TAE on QOL". All clinical information was obtained from the medial database of Toranomon Hospital.

\section{Patient population}

All patients with ADPKD who were referred to Toranomon Hospital between March 2010 and November 2012 were enrolled in this study. Patients who were less than 20 years old, patients who received renal or hepatic TAE before March 2010, and patients who did not agree to participate in this research were excluded. Patients on CAPD were also excluded because abdominal distention might be influenced by their dialysis method. All enrolled patients met the criteria for diagnosis of ADPKD as defined by Ravine et al. [15]. Some patients underwent renal or hepatic TAE after being enrolled, but others did not undergo TAE up to November 2012.
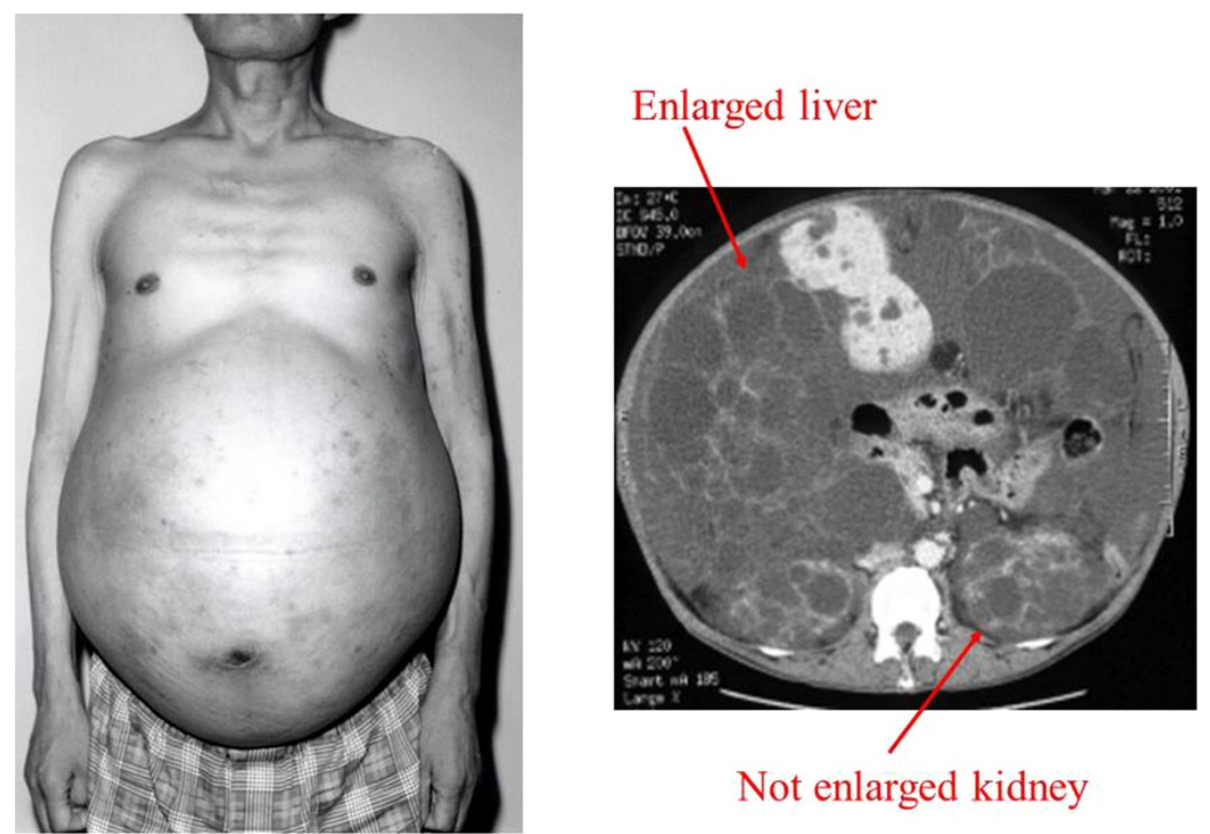

Figure $1 \mathrm{~A}$ non-dialysis ADPKD patient with massive hepatomegaly. 


\section{Assessment of QOL}

Patients completed the self-administered questionnaires during their free time. They were requested to fill in the questionnaires while they were in a normal state. All patients who underwent renal or hepatic TAE completed the questionnaires before having the procedure. The questionnaires included the Short Form-36 questionnaire (SF-36) and our 12-item original questionnaire. The SF-36 contains 36 questions that evaluate eight dimensions: physical functioning, physical role function, body pain, general health perception, vitality, social function, emotional role function and mental health. The scores for these dimensions are then totalled to obtain a physical component summary score (PCS), a mental component summary score (MCS), and a role/social component summary score (RCS). These scores have been validated in various studies of multiple chronic illnesses and have been normalized for the general Japanese population (a mean value of 50 and an SD of 10). The mean age of the general Japanese population is $50.5 \pm 15.9$ years. We also devised 12 questions about specific symptoms of patients with ADPKD, such as abdominal distension, poor appetite, and sleep disturbance (Table 1). Each symptom was assigned a score of $1-4$ or $1-5$, with 1 being the most severe.

\section{Clinical and laboratory assessments}

Height and body weight were recorded when the subjects filled in the questionnaires. Dry weight was used as the body weight of dialysis patients. Body mass index (BMI) was calculated as the weight in kilograms divided by the square of the height in meters. All patients underwent laboratory tests while they were in their usual state and within one week before or after filling in the questionnaires. The past history was investigated from the medical records of Toranomon Hospital and all associated diseases with a medical diagnosis were identified from the records. With regard to the past history, we analyzed the influence on QOL of cardiovascular disease,

\section{Table 1 Our original 12-item questionnaire}

\begin{tabular}{ll}
\hline (Q1) & Can you cut your toenails by yourself? \\
(Q2) & Can you pick up something from the floor? \\
(Q3) & Do you feel abdominal distention? \\
(Q5) & How is your appetite? \\
(Q6) & How often do you take sleeping pills? \\
(Q7) & Dou you sleep well? \\
(Q8) & How often do you take a purgative? \\
(Q9) & How often do you have constipation? \\
(Q10) & How often do you have gross hematuria? \\
(Q11) & \\
\hline
\end{tabular}

cerebrovascular disease, cancer, mental disease, and diabetes mellitus.

\section{Imaging studies}

Abdominal CT or MRI was routinely performed in all patients and images obtained within 3 months before or after entry into this study were used for evaluation of organ volumes. For all patients who received renal or hepatic TAE after being enrolled in this study, the abdominal CT or MRI studies obtained before TAE were used for evaluation of organ volume because it would have changed after TAE.

T1-weighted images (T1WI) and T2-weighted images (T2WI) obtained in the transverse and sagittal planes were employed for evaluation of organ size by MRI, which was performed with a $1.5-\mathrm{T}$ apparatus (MagnetomAvanto, Siemens, Erlangen, Germany) and a phased-array body coil. CT scanning was performed with a 16-MDCT scanner (Aquilion 16, Toshiba).

Renal volume was calculated on CT scans using the formula for an ellipsoid: $a * b * c * \pi / 6$, where $a$ is the maximum length of the kidney and $b$ and $c$ are the maximum widths in the two transverse dimensions. Each CT scan obtained at a slice interval of $1 \mathrm{~cm}$ was analyzed using Synapse software (Fujifilm Company) and the hepatic area (cysts plus parenchyma) was measured. Then the total liver volume was calculated as the sum of all the hepatic areas, while the total liver and kidney volume (TLKV) was obtained by adding total liver volume and the kidney volumes calculated as mentioned above. The existence of ascites was assessed by two different readers who were specialists in nephrology and radiology.

\section{Statistical analysis}

The mean PCS, MCS, RCS, and specific component scores were calculated for the overall study population. For quantitative discrete variables, differences between groups were assessed by using Student's $t$-test or the MannWhitney U test.

The values of PCS, MCS, and RCS were compared between the patients and the general Japanese population by the one sample $t$-test. Correlations between PCS, MCS, or RCS and TLVK were assessed by univariate analysis.

Stepwise multiple regression analysis with forward elimination was used to analyze the factors related to PCS, MCS, and RCS. These three indicators of QOL were defined as the dependent variable, while age, sex, BMI, dialysis, duration of dialysis, ascites, TLKV, serum Alb, CRP, Cr, alkaline phosphatase (ALP), cholinesterase (ChE), Hb, cardiovascular disease, cerebrovascular disease, cancer, mental disease, and diabetes mellitus were defined as the explanatory variables. The TLKV of each patient was adjusted by the body surface areafor use in all analyses.

The answers to our 12-item questionnaire were expressed on an ordinal scale and correlations with the three 
Table 2 Profile of the enrolled patients

\begin{tabular}{|c|c|c|c|c|c|c|c|c|c|c|}
\hline & $M / F$ & $\begin{array}{l}\text { Age } \\
\text { (years old) }\end{array}$ & BMI & $\begin{array}{l}\text { Duration of HD } \\
\text { (months) }\end{array}$ & $\begin{array}{l}\text { Serum Alb } \\
\text { (g/dL) }\end{array}$ & $\begin{array}{l}\text { Serum ChE } \\
\text { (IU/L) }\end{array}$ & $\begin{array}{l}\text { Serum } \mathrm{Cr} \\
(\mathrm{mg} / \mathrm{dL})\end{array}$ & $\begin{array}{l}\text { Serum CRP } \\
(\mathrm{mg} / \mathrm{dL})\end{array}$ & $\begin{array}{l}\text { Serum ALP } \\
\text { (IU/L) }\end{array}$ & $\mathrm{Hb}(\mathrm{g} / \mathrm{dL})$ \\
\hline All & $93 / 126$ & $55.1 \pm 10.8$ & $22.7 \pm 4.1$ & $74.5 \pm 61.8$ & $3.48 \pm 0.41$ & $230.8 \pm 68.6$ & $2.08 \pm 1.89$ & $0.33 \pm 0.69$ & $247.2 \pm 126.3$ & $11.48 \pm 1.71$ \\
\hline Non-dialysis & $44 / 67$ & $52.3 \pm 11.9^{*}$ & $23.4 \pm 5.0+$ & & $3.71 \pm 0.32^{*}$ & $265.8 \pm 72.5^{*}$ & $2.08 \pm 1.89$ & $0.21 \pm 0.39 \neq$ & $240.6 \pm 116.5$ & $12.22 \pm 1.72^{*}$ \\
\hline Dialysis & $49 / 59$ & $57.9 \pm 8.7^{*}$ & $21.9 \pm 2.4 \dagger$ & $74.5 \pm 61.8$ & $3.23 \pm 0.34^{*}$ & $201.2 \pm 48.6^{*}$ & & $0.45 \pm 0.88 \neq$ & $253.9 \pm 135.9$ & $10.72 \pm 1.34^{*}$ \\
\hline $\begin{array}{l}\text { No. of patients with } \\
\text { ascites (/patients available } \\
\text { abdominal image data) }\end{array}$ & Liver volume $\left(\mathrm{cm}^{3}\right)$ & $\begin{array}{l}\text { Kidney } \\
\text { volume }\left(\mathrm{cm}^{3}\right)\end{array}$ & $\begin{array}{l}\text { Total liver } \\
\text { and kidney } \\
\text { volume }\left(\mathrm{cm}^{3}\right)\end{array}$ & $\begin{array}{l}\text { No. of } \\
\text { patients with } \\
\text { cardiovascular } \\
\text { disease }\end{array}$ & $\begin{array}{l}\text { No. of } \\
\text { patients with } \\
\text { cerebrovascular } \\
\text { disease }\end{array}$ & $\begin{array}{l}\text { No. of patients } \\
\text { with cancer }\end{array}$ & $\begin{array}{l}\text { No of patients } \\
\text { with mental } \\
\text { disease }\end{array}$ & \multicolumn{3}{|c|}{ No of patients with diabetes } \\
\hline $31 / 176$ & $3573.1 \pm 3088.7$ & $3908.6 \pm 2578.3$ & $7457.2 \pm 3801.6$ & 21 & 21 & 11 & 5 & 2 & & \\
\hline $10 / 72$ & $3841.2 \pm 3550.9$ & $3308.1 \pm 2566.2^{*}$ & $7073.6 \pm 4175.2 \neq$ & 8 & 13 & 7 & 4 & 2 & & \\
\hline $21 / 104$ & $3378.2 \pm 2620.3$ & $4538.2 \pm 2487.9^{*}$ & $7935.1 \pm 3393.5 \neq$ & 13 & 8 & 4 & 1 & 0 & & \\
\hline
\end{tabular}


SF-36 scores and TLKV were assessed by Spearman's rank correlation coefficient analysis. Factor analysis was employed for multivariate analysis of the relations among the three SF-36 scores and each of our 12 questions. Promax rotation was used for factor analysis because the underlying factors were all considered to be correlated with each other to some extent. Because pain is a common symptom of ADPKD and has a detrimental influence on QOL $[8,9]$, a question about pain was included in the SF-36 and the score was used to assess the relation between pain and other SF-36 scales or TLKV by Spearman's rank correlation coefficient analysis. Factor analysis was also employed for multivariate analysis of the relations among pain and the three SF-36 scores. In all analyses, $\mathrm{P}<0.05$ was considered to indicate significance, and all analyses were performed with the SPSS Statistics 18.0 statistical software package (SPSS Inc., Chicago, IL, USA).

\section{Results}

A total of 219 patients were enrolled in this study, including 93 men and 126 women with a mean age of $55.1 \pm 10.8$ years. Among them, 108 patients were on hemodialysis (Table 2). Up to November 2012, 85 patients received renal TAE and 28 patients received hepatic TAE. Abdominal CT or MRI data for evaluation of TLKV were available in 173 patients.

The three SF-36 scores (PCS, MCS, and RCS) of all patients were significantly lower than those of the general Japanese population (Figure 2). In addition, the PCS of patients aged from their 40s to 60s and the MCS and RCS of patients aged from their 50 s to 60 s were lower than those of the general Japanese population when analysis was performed by age group (Table 3). Among all non-dialysis patients, the PCS was significantly lower than the mean value for the general Japanese population (Figure 2). The PCS of non-dialysis patients from their 40 s to 60 s was also significantly lower than that of the general Japanese population (Table 3). Comparing nondialysis patients with dialysis patients, the PCS was significantly lower for dialysis patients than non-dialysis patients, while the MCS and RCS did not differ between the two groups (Figure 2). Dialysis patients were older and had a lower BMI, lower $\mathrm{Hb}$, lower serum $\mathrm{ChE}$ and Alb levels, and higher serum CRP level than non-dialysis patients (Table 2).

Univariate analysis revealed that PCS was correlated with TLKV, though MCS and PCS were not (Figure 3). Stepwise multiple regression analysis demonstrated that $\mathrm{Hb}$ serum Alb, ascites, and cerebrovascular disease were variables with a significant influence on PCS, while mental disease was the variable significantly influencing MCS and serum Alb was the only variable influencing RCS (Table 4). TLKV and dialysis were not significantly related to any of the SF-36 scores.

Among our original questions, Q1-7, Q11, Q12, and pain were significantly correlated with PCS by Spearman's rank correlation analysis (Table 5), while Q1 and Q3 were correlated with TLKV. Thus, only Q1 and Q3 were correlated with both PCS and TLKV. According to factor analysis, PCS, Q3-5, and Q12 had high positive factor loadings, while PCS and Q11 had high negative factor loadings for the first factor (Table 6). Then PCS, Q1, and Q2 had high



Figure 2 Comparison of the SF-36 scores (PCS, MCS, and RCS) for all enrolled patients, non-dialysis patients, dialysis patients, and the general Japanese population. 
Table 3 Comparison of PCS, MCS, and RCS Comparison of PCS, MCS, and RCS between ADPKD patients and the general Japanese population

\begin{tabular}{|c|c|c|c|c|c|c|c|}
\hline Group & Item & Age group (decades) & No. of patients & Mean & SD & SEM & P value* \\
\hline \multirow[t]{15}{*}{ All patients } & \multirow[t]{5}{*}{ PCS } & $30 \mathrm{~s}$ & 17 & 52.1 & 7.6 & 1.9 & NS \\
\hline & & $40 \mathrm{~s}$ & 52 & 44.2 & 13.4 & 1.9 & $<0.001$ \\
\hline & & $50 \mathrm{~s}$ & 73 & 41.8 & 11.6 & 1.4 & $<0.001$ \\
\hline & & $60 \mathrm{~s}$ & 58 & 36.2 & 14.9 & 2.0 & $<0.001$ \\
\hline & & $70 \mathrm{~s}$ & 19 & 41.2 & 11.7 & 2.7 & NS \\
\hline & \multirow[t]{5}{*}{ MCS } & $30 \mathrm{~s}$ & 17 & 52.7 & 10.5 & 2.5 & NS \\
\hline & & $40 \mathrm{~s}$ & 52 & 46.7 & 9.5 & 1.3 & NS \\
\hline & & $50 \mathrm{~s}$ & 73 & 47.6 & 7.7 & 0.9 & $<0.05$ \\
\hline & & $60 \mathrm{~s}$ & 58 & 45.9 & 10.5 & 1.4 & $<0.001$ \\
\hline & & $70 \mathrm{~s}$ & 19 & 55.7 & 8.2 & 1.9 & NS \\
\hline & \multirow[t]{5}{*}{ RCS } & $30 \mathrm{~s}$ & 17 & 50.1 & 12.5 & 3.0 & NS \\
\hline & & $40 \mathrm{~s}$ & 52 & 47.6 & 15.5 & 2.2 & NS \\
\hline & & $50 \mathrm{~s}$ & 73 & 45.8 & 14.6 & 1.7 & $<0.001$ \\
\hline & & $60 \mathrm{~s}$ & 58 & 46.0 & 17.3 & 2.3 & $<0.01$ \\
\hline & & $70 \mathrm{~s}$ & 19 & 44.8 & 13.8 & 3.2 & NS \\
\hline \multirow[t]{15}{*}{ Non-dialysis patients } & \multirow[t]{5}{*}{ PCS } & $30 \mathrm{~s}$ & 15 & 52.7 & 7.9 & 2.1 & NS \\
\hline & & $40 \mathrm{~s}$ & 34 & 47.6 & 13.0 & 2.2 & $<0.05$ \\
\hline & & $50 \mathrm{~s}$ & 29 & 44.7 & 11.1 & 2.1 & $<0.05$ \\
\hline & & $60 \mathrm{~s}$ & 23 & 37.9 & 17.3 & 3.6 & 0.001 \\
\hline & & $70 \mathrm{~s}$ & 10 & 43.4 & 11.8 & 3.7 & NS \\
\hline & \multirow[t]{5}{*}{ MCS } & $30 \mathrm{~s}$ & 15 & 52.2 & 11.0 & 2.8 & NS \\
\hline & & $40 \mathrm{~s}$ & 34 & 47.0 & 10.2 & 1.7 & NS \\
\hline & & $50 \mathrm{~s}$ & 29 & 45.6 & 7.4 & 1.4 & $<0.01$ \\
\hline & & $60 \mathrm{~s}$ & 23 & 48.4 & 11.0 & 2.3 & 0.05 \\
\hline & & $70 \mathrm{~s}$ & 10 & 57.4 & 4.5 & 1.4 & NS \\
\hline & \multirow[t]{5}{*}{ RCS } & $30 \mathrm{~s}$ & 15 & 52.1 & 8.5 & 2.2 & NS \\
\hline & & $40 \mathrm{~s}$ & 34 & 47.9 & 14.0 & 2.4 & NS \\
\hline & & $50 \mathrm{~s}$ & 29 & 47.5 & 14.0 & 2.6 & 0.01 \\
\hline & & $60 \mathrm{~s}$ & 23 & 49.5 & 13.4 & 2.8 & NS \\
\hline & & $70 \mathrm{~s}$ & 10 & 50.1 & 11.9 & 3.8 & NS \\
\hline \multirow[t]{10}{*}{ Dialysis patients } & \multirow[t]{5}{*}{ PCS } & $30 \mathrm{~s}$ & 2 & 47.5 & 0.6 & 0.5 & NS \\
\hline & & $40 \mathrm{~s}$ & 18 & 37.7 & 11.9 & 2.8 & $<0.001$ \\
\hline & & $50 \mathrm{~s}$ & 44 & 40.0 & 11.7 & 1.8 & $<0.001$ \\
\hline & & $60 \mathrm{~s}$ & 35 & 35.1 & 13.2 & 2.2 & $<0.001$ \\
\hline & & $70 \mathrm{~s}$ & 9 & 38.8 & 11.8 & 3.9 & NS \\
\hline & \multirow[t]{5}{*}{ MCS } & $30 \mathrm{~s}$ & 2 & 56.3 & 6.1 & 4.3 & NS \\
\hline & & $40 \mathrm{~s}$ & 18 & 45.9 & 8.2 & 1.9 & NS \\
\hline & & $50 \mathrm{~s}$ & 44 & 49.0 & 7.6 & 1.2 & NS \\
\hline & & $60 \mathrm{~s}$ & 35 & 44.2 & 9.9 & 1.7 & $<0.001$ \\
\hline & & $70 \mathrm{~s}$ & 9 & 53.8 & 10.9 & 3.6 & NS \\
\hline
\end{tabular}


Table 3 Comparison of PCS, MCS, and RCS Comparison of PCS, MCS, and RCS between ADPKD patients and the general Japanese population (Continued)

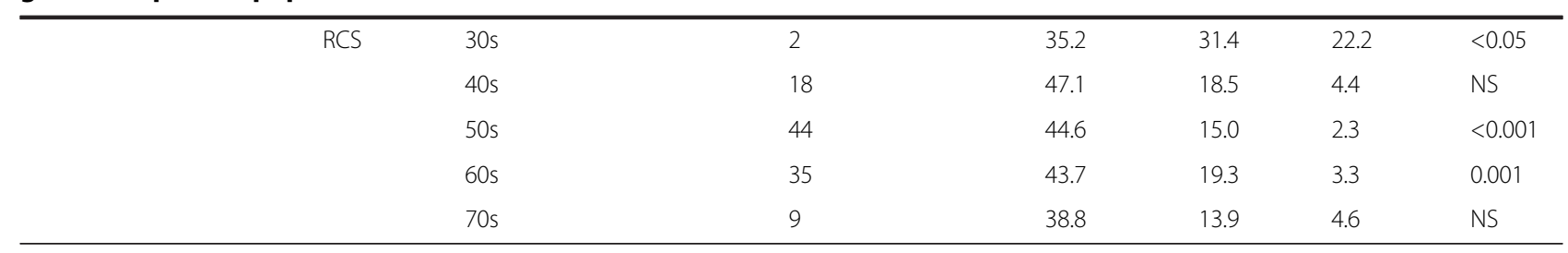

* Comparison with the data of general Japanese population was analyzed in each age group.

SD: Standard Deviation, SE: standard error of the mean, NS: not significant.

positive factor loadings for the second factor. Because the second factor has higher positive factor loadings than the first factor, Q1 and Q2 (with high positive loadings for the second factor) were considered to be better correlated with PCS than Q3. Since Q3-5, Q7, Q12, and pain were significantly correlated with MCS, Q3 was the only question correlated with both MCS and TLKV (Table 5). Factor analysis also showed that MCS, Q3-5, and Q12 had high positive factor loadings, while MCS and pain had high negative factor loadings for the first factor (Table 7). Since Q1-4, Q6, Q11, and pain were significantly correlated with RCS, only Q1 and Q3 were correlated with both RCS and TLKV (Table 5). Furthermore, RCS, Q7, and Q11 had high positive factor loadings, while RCS, Q5, and pain had high negative factor loadings for the second factor (Table 8).

\section{Discussion}

Rizk et al. reported that the SF-36 score of non-dialysis ADPKD patients was not significantly different from that of the general population. In the present study, however, the PCS of non-dialysis ADPKD patients was significantly lower than that of the general Japanese population. Our analysis stratified for age groups also showed

PCS $=44.9-8.95 \times 10^{-4} \times$ TLKV (adjusted by each body surface area) $R^{2}=0.027 \quad P=0.032 \quad \beta=-0.164$

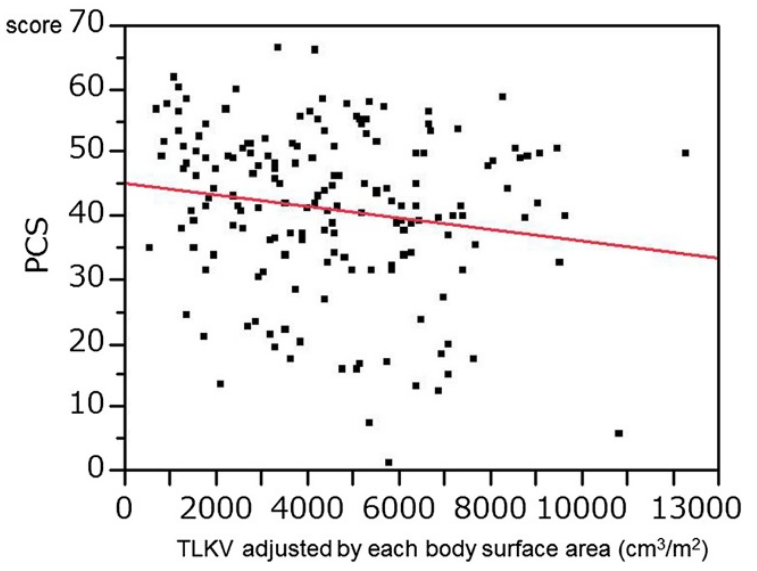

Figure 3 Univariate regression analysis of the relation between PCS and TLKV. that the PCS of non-dialysis patients from their 40s to 60 s was significantly lower than that of the general Japanese population. This difference from the findings of Rizk et al. may have occurred because the mean kidney volume of the non-dialysis patients in our study was much larger than that of the patients they investigated. PCS was significantly lower in dialysis patients than non-dialysis patients, but age, nutritional status, and kidney volume were significantly different between the two groups.

It was reasonable that ascites and cerebrovascular disease were variables with a significant influence on PCS and mental disease was significantly associated with MCS. Serum Alb might reflect the nutritional status which is influenced by multiple factors, including the appetite, liver function, renal function, TLKV, and lifestyle. Then, it might not doubtful that Alb was significant variable for PCS and RCS. It might be the same reason for association $\mathrm{Hb}$ and $\mathrm{PCS}$.

TLKV and dialysis were not significantly associated with any of the SF-36 scores by stepwise multiple regression analysis. However, univariate analysis revealed that PCS was correlated with TLKV, so TLKV seems to have an important influence on PCS. It seems that PCS was not only affected by abdominal distention (strongly correlated with TLKV), but also by several symptoms unrelated to TLKV. Accordingly, there should be multiple factors that influence the QOL of ADPKD in addition to TLKV, which is one of the reasons that TLKV was not

Table 4 Stepwise multiple logistic regression analysis of each SF-36 score

\begin{tabular}{lllll}
\hline $\begin{array}{l}\text { Dependent } \\
\text { variable }\end{array}$ & $\begin{array}{l}\text { Explanatory } \\
\text { variable }\end{array}$ & $\boldsymbol{\beta}$ & P value & Adjusted R \\
\hline PCS & Alb & 0.223 & $<0.01$ & 0.187 \\
& $\mathrm{Hb}$ & 0.161 & $<0.05$ & \\
& Ascites & -0.214 & $<0.005$ & \\
& Cerebrovascular disease & -0.193 & $<0.01$ & \\
\hline MCS & mental disease & -0.171 & $<0.05$ & 0.023 \\
\hline RCS & Alb & 0.247 & 0.001 & 0.055
\end{tabular}

$\beta$ : Standardized regression coefficient

Variables included in the model were age, sex, BMI, dialysis, dialysis duration, ascites, TLKV, Alb, CRP, Cr, ALP, ChE, Hb, cardiovascular disease,

cerebrovascular disease, cancer, mental disease, and diabetes. 
Table 5 Spearman's rank correlation coefficient (original questions vs. three SF-36 scores, and original questions vs. TLKV)

\begin{tabular}{|c|c|c|c|c|c|c|c|c|}
\hline \multirow[b]{2}{*}{ Question } & \multicolumn{2}{|c|}{ PCS } & \multicolumn{2}{|c|}{ MCS } & \multicolumn{2}{|c|}{ RCS } & \multicolumn{2}{|c|}{ TLKV } \\
\hline & $\overline{r_{s}}$ & $p$ & $\overline{r_{s}}$ & $P$ & $\overline{r_{s}}$ & $P$ & $\overline{r_{s}}$ & $P$ \\
\hline $\mathrm{Q1}$ & 0.313 & $<0.001$ & 0.127 & NS & 0.198 & $<0.005$ & -0.189 & $<0.05$ \\
\hline Q2 & 0.366 & $<0.001$ & 0.090 & NS & 0.337 & $<0.001$ & -0.148 & NS \\
\hline Q3 & 0.450 & $<0.001$ & 0.357 & $<0.001$ & 0.306 & $<0.001$ & -0.328 & $<0.001$ \\
\hline Q4 & 0.235 & 0.001 & 0.388 & $<0.001$ & 0.210 & $<0.005$ & 0.044 & NS \\
\hline Q5 & 0.274 & $<0.001$ & 0.379 & $<0.001$ & 0.133 & NS & -0.092 & NS \\
\hline Q6 & 0.189 & $<0.05$ & 0.135 & NS & 0.179 & $<0.05$ & 0.066 & NS \\
\hline Q7 & 0.290 & $<0.001$ & 0.267 & $<0.001$ & 0.126 & NS & -0.072 & NS \\
\hline Q8 & -0.009 & NS & 0.084 & NS & -0.009 & NS & 0.077 & NS \\
\hline Q9 & 0.116 & NS & 0.037 & NS & 0.003 & NS & -0.148 & NS \\
\hline Q10 & 0.007 & NS & 0.010 & NS & 0.004 & NS & 0.117 & NS \\
\hline Q11 & 0.162 & $<0.05$ & 0.095 & NS & 0.163 & $<0.05$ & -0.030 & NS \\
\hline Q12 & 0.266 & $<0.001$ & 0.163 & $<0.05$ & 0.017 & NS & -0.077 & NS \\
\hline Pain & -0.582 & $<0.001$ & -0.437 & $<0.001$ & -0.193 & $<0.005$ & -0.083 & NS \\
\hline
\end{tabular}

$\mathrm{r}_{\mathrm{s}}$ : Spearman's rank correlation coefficient, NS: not significant.

significantly associated with PCS in multiple regression analysis. In addition, factor analysis revealed that Q1 and Q2 were more closely associated with PCS than Q3. Thus, the ability to cut toenails or to pick up something from the floor affected PCS more than abdominal distention.

Table 6 Results of factor analysis of the PCS: Promax (oblique) rotated factor patterns

\begin{tabular}{|c|c|c|c|c|c|}
\hline & 1 & II & III & IV & V \\
\hline PCS & 0.360 & 0.633 & 0.052 & 0.046 & -0.059 \\
\hline Q1 & -0.065 & 0.825 & -0.012 & 0.041 & -0.082 \\
\hline Q2 & -0.045 & 0.847 & 0.135 & -0.054 & -0.006 \\
\hline Q3 & 0.739 & 0.273 & -0.086 & 0.064 & 0.113 \\
\hline Q4 & 0.757 & -0.122 & 0.191 & -0.226 & -0.112 \\
\hline Q5 & 0.843 & -0.082 & -0.309 & 0.015 & -0.048 \\
\hline Q6 & 0.129 & -0.109 & -0.001 & 0.728 & 0.229 \\
\hline Q7 & 0.192 & 0.053 & 0.584 & -0.124 & 0.024 \\
\hline Q8 & 0.007 & -0.162 & 0.261 & 0.083 & 0.632 \\
\hline Q9 & -0.214 & 0.145 & -0.076 & 0.797 & -0.176 \\
\hline Q10 & 0.051 & -0.006 & 0.132 & 0.033 & -0.805 \\
\hline Q11 & -0.328 & 0.149 & 0.915 & 0.001 & 0.057 \\
\hline Q12 & 0.327 & -0.269 & 0.380 & 0.290 & -0.198 \\
\hline pain & -0.495 & -0.287 & -0.127 & -0.076 & -0.127 \\
\hline \multicolumn{6}{|c|}{ Inter-factor correlations } \\
\hline । & - & 0.225 & 0.424 & 0.206 & 0.037 \\
\hline$\|$ & 0.225 & - & 0.159 & 0.095 & -0.024 \\
\hline III & 0.424 & 0.159 & - & 0.173 & 0.025 \\
\hline IV & 0.206 & 0.095 & 0.173 & - & -0.074 \\
\hline V & 0.037 & -0.024 & 0.025 & -0.074 & - \\
\hline
\end{tabular}

These abilities might be associated with muscular strength and balance as well as abdominal distension.

TLKV was also not significantly associated with MCS on multiple regression analysis. One of the reasons was that mental disease was a significant variable, but our

Table 7 Results of factor analysis of the MCS: Promax (oblique) rotated factor patterns

\begin{tabular}{|c|c|c|c|c|c|}
\hline & 1 & II & III & IV & V \\
\hline MCS & 0.776 & -0.194 & -0.070 & 0.017 & 0.130 \\
\hline Q1 & 0.059 & 0.829 & -0.026 & 0.077 & -0.066 \\
\hline Q2 & 0.020 & 0.858 & 0.200 & -0.037 & -0.001 \\
\hline Q3 & 0.729 & 0.312 & -0.056 & 0.089 & 0.066 \\
\hline Q4 & 0.721 & -0.052 & 0.178 & -0.222 & -0.162 \\
\hline Q5 & 0.762 & 0.021 & -0.243 & 0.014 & -0.109 \\
\hline Q6 & 0.161 & -0.084 & -0.004 & 0.733 & 0.214 \\
\hline Q7 & 0.253 & 0.058 & 0.503 & -0.107 & 0.015 \\
\hline Q8 & -0.070 & -0.139 & 0.398 & 0.053 & 0.563 \\
\hline Q9 & -0.195 & 0.144 & -0.056 & 0.792 & -0.175 \\
\hline Q10 & -0.023 & -0.007 & 0.140 & 0.011 & -0.825 \\
\hline Q11 & -0.246 & 0.165 & 0.891 & -0.027 & 0.045 \\
\hline Q12 & 0.248 & -0.262 & 0.429 & 0.259 & -0.263 \\
\hline pain & -0.531 & -0.214 & -0.200 & -0.062 & -0.121 \\
\hline \multicolumn{6}{|c|}{ Inter-factor correlations } \\
\hline I & - & 0.063 & 0.379 & 0.168 & 0.015 \\
\hline$\|$ & 0.063 & - & 0.082 & 0.027 & -0.060 \\
\hline III & 0.379 & 0.082 & - & 0.143 & -0.005 \\
\hline IV & 0.168 & 0.027 & 0.143 & - & -0.081 \\
\hline V & 0.015 & -0.060 & -0.005 & -0.081 & - \\
\hline
\end{tabular}


Table 8 Results of factor analysis of the RCS: Promax (oblique) rotated factor patterns

\begin{tabular}{lllllll}
\hline & I & II & III & IV & V & VI \\
\hline RCS & 0.097 & 0.644 & 0.077 & 0.093 & 0.254 & -0.223 \\
Q1 & 0.103 & -0.073 & 0.864 & 0.033 & -0.054 & -0.017 \\
Q2 & 0.085 & 0.103 & 0.858 & -0.083 & -0.034 & 0.082 \\
Q3 & 0.736 & 0.087 & 0.204 & 0.101 & 0.118 & -0.023 \\
Q4 & 0.678 & 0.294 & -0.146 & -0.201 & -0.153 & -0.086 \\
Q5 & 0.848 & -0.305 & 0.082 & -0.026 & -0.088 & 0.109 \\
Q6 & 0.119 & 0.069 & -0.193 & 0.760 & 0.155 & 0.043 \\
Q7 & 0.130 & 0.680 & -0.142 & -0.048 & -0.031 & -0.122 \\
Q8 & 0.050 & -0.084 & 0.052 & -0.059 & 0.230 & 0.944 \\
Q9 & -0.183 & -0.082 & 0.145 & 0.789 & -0.169 & -0.122 \\
Q10 & 0.026 & -0.049 & 0.106 & -0.035 & -0.860 & -0.291 \\
Q11 & -0.356 & 0.832 & 0.133 & -0.030 & -0.125 & 0.227 \\
Q12 & 0.270 & 0.120 & -0.142 & 0.192 & -0.494 & 0.301 \\
pain & -0.403 & -0.328 & -0.131 & -0.074 & -0.043 & -0.053 \\
\hline Inter-factor correlations & & & & \\
I & - & 0.381 & 0.073 & 0.173 & -0.051 & 0.091 \\
II & 0.381 & - & 0.218 & 0.146 & -0.069 & 0.186 \\
III & 0.073 & 0.218 & - & 0.101 & 0.020 & -0.112 \\
IV & 0.173 & 0.146 & 0.101 & - & -0.098 & 0.084 \\
V & -0.051 & -0.069 & 0.020 & -0.098 & - & -0.183 \\
VI & 0.091 & 0.186 & -0.112 & 0.084 & -0.183 & - \\
\hline
\end{tabular}

12-item questionnaire did not have any questions associated directly with mental disease. Symptoms associated with mental disease might have had higher positive factor loadings than abdominal distention. There was a weaker correlation between RCS and abdominal distension according factor analysis and Spearman's rank correlation analysis. RCS might be more strongly affected by the social and family environment of each patient, but questions about the family and social environment were not included in this study.

As reported before, pain was an important factor for QOL because it was correlated with all of the SF-36 scores according to Spearman's rank correlation analysis and factor analysis [8,9]. Sleep disturbance, heartburn, fever, gross hematuria and anorexia were also important factors for QOL, while snoring and constipation were not important factors. Though it is doubtful that gross hematuria influenced QOL, it might be related to fever and pain. Although pain, sleep disturbance, heartburn, fever, gross hematuria, and anorexia were all important for QOL, these variables were not correlated with TLKV. Thus, improving these factors unrelated to TLKV is considered to be important for elevating the QOL of ADPKD patients as well as reduction of abdominal distention.
Against our expectations, TLKV did not have a very strong influence on the QOL of ADPKD patients, although it was considered to have some influence on QOL. In order to reveal how TLKV affect SF-36 more clearly, we would need to study the changes of TLKV in each patient over time. Such a study about the changes of QOL in ADPKD patients after renal TAE is now ongoing and it will be able to reveal how SF-36 scores change when kidney volume is dramatically reduced by renal TAE. SF-36 did not reflect sensitively TLKV or abdominal distention in this study, so evaluation using SF-36 alone might not be suitable for assessing the symptoms of ADPKD patients. We will continue to use our original questions as well.

Against our expectation, dialysis did not have a significant influence on any of the SF-36 scores. One reason might be that QOL measured by SF-36 includes both symptoms affected by dialysis and symptoms unrelated to dialysis. In addition, the environment for dialysis patients (including techniques and medical expenses) is well developed in Japan and there are almost 20,000 patients who have been on hemodialysis for longer than 20 years [16]. This might have affected our results as well.

The limitations of this study are that it was based on a questionnaire and each answer was subjective. Also, only Japanese patients were included in this study, and there might be difference among ethnic groups and dialysis conditions in Japan might be different from those elsewhere in the world. There might also be other confounding factors that affect the QOL of ADPKD patients, but familial and social factors were not included in this study. Finally, the QOL of patients with ADPKD was mainly assessed with the SF-36 in this study, but it is uncertain how accurately this questionnaire reveals the actual situation of ADPKD patients.

\section{Conclusion}

This study demonstrated that the mean PCS, MCS, and RCS of all patients were significantly lower than those of the general Japanese population. There was a significant difference of PCS between dialysis and non-dialysis patients, despite no significant differences of MCS and RCS. Even the PCS of non-dialysis patients was significantly lower than that of the general Japanese population. Stepwise multiple regression analysis demonstrated that $\mathrm{Hb}$, serum Alb, ascites, and cerebrovascular disease were variables with a significant influence on PCS, while mental disease was the only significant factor for MCS and serum Alb was the only significant factor for RCS. TLVK and dialysis did not have a significant influence on any of the SF36 scores according to stepwise multiple regression analysis. However, TLKV was well correlated with abdominal distention and abdominal distention was an important factor for QOL. Pain, sleep disturbance, heartburn, fever, gross hematuria, and anorexia were also important factors for QOL, 
but were not correlated with TLKV. Thus, QOL was considered to be a multifactorial construct and addressing symptoms unrelated to TLKV as well as abdominal distention is important for improving the QOL of ADPKD patients.

\section{Abbreviations}

QOL: Quality of life; ADPKD: Autosomal dominant polycystic kidney disease; SF-36: The short form-36; TAE: Transcatheter arterial embolization; TLKV: Total liver and kidney volume; PCS: Physical component summary score; MCS: Mental component summary score; RCS: Role/social component summary score (RCS).

\section{Competing interests}

All the authors declared no competing interest.

\section{Authors' contributions}

All co-authors carried out questionnaire survey on their patients, and collected questionnaires. YU conceived of the study, and participated in its design and coordination and helped to draft the manuscript. KT responsible person and participated in the design of the study. All co-authors read and approved the final manuscript.

\section{Acknowledgements}

This study was supported by funding in Toranomon Hospital. Analysis and interpretation of data in this study was supported by Satista Co., Ltd. The English in this document has been checked by native speakers of English in Yamada Translation Bureau. Inc.

Received: 20 February 2013 Accepted: 23 August 2013

Published: 27 August 2013

\section{References}

1. Davies F, Coles GA, Harper PS, Williams AJ, Evans C, Cochlin D: Polycystic kidney disease re-evaluated: a population-based study. Q J Med 1991, 79(290):477-485.

2. Gabow PA: Autosomal dominant polycystic kidney disease. N Engl J Med 1993, 329(5):332-342.

3. Ubara Y, Tagami T, Sawa N, Katori H, Yokota M, Takemoto F, Inoue S, Kuzuhara K, Hara S, Yamada A: Renal contraction therapy for enlarged polycystic kidneys by transcatheter arterial embolization in hemodialysis patients. Am J Kidney Dis 2002, 39(3):571-579.

4. Ubara Y, Takei R, Hoshino J, Tagami T, Sawa N, Yokota M, Katori H, Takemoto F, Hara S, Takaichi K: Intravascular embolization therapy in a patient with an enlarged polycystic liver. Am J Kidney Dis 2004 , 43(4):733-738.

5. Ubara Y: New therapeutic option for autosomal dominant polycystic kidney disease patients with enlarged kidney and liver. TherApher Dial 2006, 10(4):333-341.

6. Takei R, Ubara Y, Hoshino J, Higa Y, Suwabe T, Sogawa Y, Nomura K, Nakanishi S, Sawa N, Katori H, Takemoto F, Hara S, Takaichi K: Percutaneous transcatheter hepatic artery embolization for liver cysts in autosomal dominant polycystic kidney disease. Am J Kidney Dis 2007, 49(6):744-752.

7. Levenson $\mathrm{J}$, Glocheski S: Psychological factors affecting end-stage renal disease. Psychosomatics 1991, 32(4):382-389.

8. Bajwa ZH, Gupta S, Warfield CA, Steinman TI: Pain management in polycystic kidney disease. Kidney int 2001, 60(5):1631-1644

9. Heiwe S, Bjuke M: "An evil heritage": interview study of pain and autosomal dominant polycystic kidney disease. Pain Manag Nurs 2009, 10(3):134-141

10. Torres VE, Chapman AB, Devuyst O, Gansevoort RT, Grantham JJ, Higashihara E, Perrone RD, Krasa HB, Ouyang J, Czerwiec FS: TEMPO 3:4 Trial investigators: tolvaptan in patients with autosomal dominant polycystic kidney disease. N Engl J Med 2012, 367(25):2407-2418.

11. Rizk D, Jurkovitz C, Veledar E, Bagby S, Baumgarten DA, Rahbari-Oskoui F, Steinman T, Chapman AB: Quality of life in autosomal dominant polycystic kidney disease patients not yet on dialysis. Clin J Am SoC Nephrol 2009, 4(3):560-566
12. Fukuhara S, Bito S, Green J, Hsiao A, Kurokawa K: Translation, adaptation, and validation of the SF-36 Health Survey for use in Japan. J Clin Epidemiol 1998, 51(11):1037-1044.

13. Fukuhara S, Ware JE Jr, Kosinski M, Wada S, Gandek B: Psychometric and clinical tests of validity of the Japanese SF-36 Health Survey. J Clin Epidemiol 1998, 51(11):1045-1053.

14. Fukuhara S, Suzukamo Y: Manual of SF-36v2 Japanese version. Kyoto: Institute for Health Outcomes \& Process Evaluation research; 2004.

15. Ravine D, Gibson RN, Walker RG, Sheffield L, Kincaid-Smith P, Danks DM: Evaluation of ultrasonographic diagnostic criteria for autosomal dominant polycystic kidney disease 1. Lancet 1994, 343(8901):824-827.

16. Akizawa T: Current status of dialysis therapy and related clinical guidelines in Japan. Japan Medical Association Journal 2010, 53(3):185-187.

doi:10.1186/1471-2369-14-179

Cite this article as: Suwabe et al:: Quality of life of patients with ADPKD -Toranomon PKD QOL study: cross-sectional study. BMC Nephrology 2013 14:179.

\section{Submit your next manuscript to BioMed Central and take full advantage of:}

- Convenient online submission

- Thorough peer review

- No space constraints or color figure charges

- Immediate publication on acceptance

- Inclusion in PubMed, CAS, Scopus and Google Scholar

- Research which is freely available for redistribution 